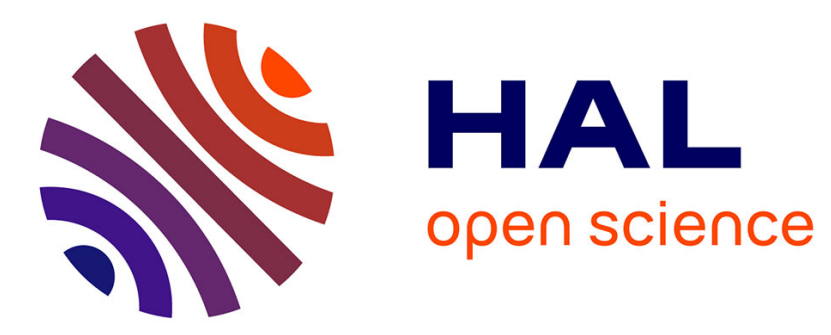

\title{
Continuous Belief Functions: Focal Intervals Properties.
}

\author{
Jean-Marc Vannobel
}

\section{To cite this version:}

Jean-Marc Vannobel. Continuous Belief Functions: Focal Intervals Properties.. BELIEF 2012, May 2012, Compiègne, France. pp.93-100. hal-00766008

\section{HAL Id: hal-00766008 https://hal.science/hal-00766008}

Submitted on 17 Dec 2012

HAL is a multi-disciplinary open access archive for the deposit and dissemination of scientific research documents, whether they are published or not. The documents may come from teaching and research institutions in France or abroad, or from public or private research centers.
L'archive ouverte pluridisciplinaire HAL, est destinée au dépôt et à la diffusion de documents scientifiques de niveau recherche, publiés ou non, émanant des établissements d'enseignement et de recherche français ou étrangers, des laboratoires publics ou privés. 


\title{
Continuous belief functions: focal intervals properties
}

Jean-Marc Vannobel

\begin{abstract}
The set of focal elements resulting from a conjunctive or disjunctive combination of consonant belief functions is regretfully not consonant and is thus very difficult to represent.

In this paper, we propose a graphical representation of the cross product of two focal sets originating from univariate Gaussian pdfs. This representation allows to represent initial focal intervals as well as focal intervals resulting from a combination operation. We show in case of conjunctive or disjunctive combination operations, that the whole domain can be separated in four subsets of intervals having same properties. At last, we focus on identical length focal intervals resulting from a combination. We show that such intervals are organized in connected line segments on our graphical representation.
\end{abstract}

\section{Introduction}

\subsection{Sources of information}

Consider a source of information $\mathcal{S}_{i}$ with knowledge modeled by a univariate convex (unimodal and consonant) probability density function bet $f_{i}$ of a continuous random variable $X$. The support of bet $f_{i}$ is called $\Omega_{i}=\left[\Omega_{i}^{-}, \Omega_{i}^{+}\right]$ with $\Omega_{i}^{-}, \Omega_{i}^{+} \in \mathcal{R}[4]$. The mode and the variance of bet $f_{i}$ are respectively noted $\mu_{i}$ and $\sigma_{i}^{2}$. Suppose now $\mathcal{E}_{i}=\left(\mathcal{F}_{i}, m_{i}\right)$, the piece of evidence deduced from bet $f_{i} . \mathcal{E}_{i}$ is totally described by the pair composed of $m_{i}$, the Least Commited isopignistic basic belief density (bbd) deduced from bet $f_{i}$ [1] [4] and $\mathcal{F}_{i}=\left\{I \subseteq \Omega_{i} \mid m_{i}(I)>0\right\}$, the focal set of intervals with elements in $\Omega_{i}$.

Jean-Marc Vannobel

LAGIS, Université Lille1, e-mail: jean-marc.vannobel@univ-lillel.fr 


\subsection{Focal intervals}

An interval $A=\left[A^{-}, A^{+}\right]$with $A^{-}, A^{+} \in \mathcal{R}$ such as $m_{i}(A) \neq 0$ is called focal interval of $\mathcal{E}_{i}$ thus $A \in \mathcal{F}_{i}$. All elements of $\mathcal{F}_{i}$ are nested intervals in case of a consonant pdf bet $f_{i}$ and correspond to horizontal cuts of bet $f_{i}$ as shown in figure $1(\mathrm{a})$. It is convenient to label the elements of $\mathcal{F}_{i}$ according to their inclusion order by a continuous index. This can be done for instance wrt the pdf value at focal interval bounds [2] or wrt the half-length of the focal interval [3]. This last option allows in general to define a single bbd's expression for a whole family of pdfs [6]. In case of symmetrical pdfs like Gaussian ones as well as Laplace ones, focal intervals can be labeled by an index $z$ such as $A^{z}=\left[A^{z-}, A^{z+}\right]$ with:

$$
\begin{gathered}
z=\frac{|x-\mu|}{\sigma}, z \in \mathcal{R}^{+}, \\
A^{z-}=\mu-\sigma z, A^{z-} \in\left[\Omega^{-}, \mu\right], \\
A^{z+}=\mu+\sigma z, A^{z+} \in\left[\mu, \Omega^{+}\right] .
\end{gathered}
$$

\section{Focal sets graphical representations}

\subsection{General considerations}

We consider in what follows two pieces of evidence $\mathcal{E}_{i}=\left(\mathcal{F}_{i}, m_{i}\right)$ and $\mathcal{E}_{j}=$ $\left(\mathcal{F}_{j}, m_{j}\right)$ deduced respectively from the Gaussian pdfs betf $f_{i}\left(x ; \mu_{i}, \sigma_{i}^{2}\right)$ and bet $f_{j}\left(x ; \mu_{j}, \sigma_{j}^{2}\right)$ with $\mu_{i} \leq \mu_{j}$. Focal intervals are denoted by $A_{i}^{z_{k}}$ with $z_{k}$ the value taken by $z_{i}$ the label obtained using relation (1). We assume the use of Gaussian pdfs since many sensors model uncertainty by such pdfs but any other symmetrical bell shaped pdfs like Laplace or Cauchy ones would also serve the purpose. $\mathcal{F}_{i, j}$ is the focal set resulting from a conjunctive (resp. disjunctive) combination of $\mathcal{E}_{i}$ and $\mathcal{E}_{j}$. Elements of $\mathcal{F}_{i, j}$ correspond to the non empty intersection (resp. union) of pairs in $\mathcal{F}_{i} \times \mathcal{F}_{j}$. The content of $\mathcal{F}_{i, j}$ and the length dependencies between its elements depend of course on the chosen combination rule.

\subsection{Bell shaped probability density functions}

The graphical representation proposed in figure $1\left(\right.$ a) shows the focal set $\mathcal{F}_{i}$ obtained from a Gaussian pdf $\operatorname{Bet} f_{i}$. Elements of $\mathcal{F}_{i}$ are ordered wrt label $z$, differing in that point from the graphical representation proposed by Strat [5]. 
When labeling focal intervals wrt their length as defined in (1), the focal set $\mathcal{F}_{i}$ is encompassed by two symmetrical half-lines defining an isosceles triangle. Equations of these half-lines are deduced from relations (2) and (3) and correspond to:

$$
\left\{\begin{array}{l}
z=\frac{\left|A_{i}^{z-}-\mu_{i}\right|}{\sigma_{i}}, \\
z=\frac{\left|A_{i}^{z+}-\mu_{i}\right|}{\sigma_{i}} .
\end{array}\right.
$$

As shown in figure 1(b), this is a convenient way to graphically compare focal intervals coming from different focal sets. One can see in this figure the result of the intersection or union of two intervals $A_{i}^{z_{k}} \in \mathcal{F}_{i}$ and $B_{j}^{z_{l}} \in \mathcal{F}_{j}$ which are indexed resp. by $z_{k}$ and $z_{l}$. For instance, it also allows to show the domain of intervals $B_{j} \in \mathcal{F}_{j}$ that do not intersect with $A_{i}^{z_{k}}$ (if any). It is obvious from relation (1) that the label value of these $B_{j}$ intervals is in $\left[0, \frac{\left|A_{i}^{z_{k}+}-\mu_{j}\right|}{\sigma_{j}}\right)$.

\subsection{Bounds relations of $\mathcal{F}_{i} \times \mathcal{F}_{j}$ elements}

Figure 2(a) shows the pairs of intervals $\left(A_{i}^{z_{i}^{x}}, B_{j}^{z_{j}^{x}}\right) \in \mathcal{F}_{i} \times \mathcal{F}_{j}$ having a common bound $x \in \Omega$. The index pairs $\left(z_{i}^{x}, z_{j}^{x}\right) \in \mathcal{R}^{+2}$ corresponding to $\left(A_{i}^{z_{i}^{x}}, B_{j}^{z_{j}^{x}}\right)$ draw the lines (1), (2) and (3) as shown in Figure 2(a). These lines are defined by $^{1}$ :

$$
\begin{cases}\text { (1) }: z_{j}^{x}=\frac{\left|\mu_{i}-\mu_{j}\right|}{\sigma_{j}}+\frac{\sigma_{i}}{\sigma_{j}} z_{i}^{x}, & x \in\left[-\infty, \mu_{i}\right], \\ \text { (2) }: z_{j}^{x}=\frac{\left|\mu_{i}-\mu_{j}\right|}{\sigma_{j}}-\frac{\sigma_{i}}{\sigma_{j}} z_{i}^{x}, \quad x \in\left[\mu_{i}, \mu_{j}\right], \\ \text { (3) }: z_{j}^{x}=\frac{-\left|\mu_{i}-\mu_{j}\right|}{\sigma_{j}}+\frac{\sigma_{i}}{\sigma_{j}} z_{i}^{x}, x \in\left[\mu_{j},+\infty\right] .\end{cases}
$$

Pairs $\left(z_{i}^{x}, z_{j}^{x}\right)$ on the half line called (1) lead to $x \leq \mu_{i}$ such as:

$$
\left\{\begin{array}{l}
A_{i}^{z_{i}^{x}-}=B_{j}^{z_{j}^{x}-} \\
A_{i}^{z_{i}^{x}+} \leq B_{j}^{z_{j}^{x}+}
\end{array}\right.
$$

Pairs $\left(z_{i}^{x}, z_{j}^{x}\right)$ on line segment (2) correspond to $x \in\left[\mu_{i}, \mu_{j}\right]$ such as:

$$
A_{i}^{z_{i}^{x}+}=B_{j}^{z_{j}^{x}-}=x
$$

At last, pairs $\left(z_{i}^{x}, z_{j}^{x}\right)$ on the half line (3) correspond to $x \geq \mu_{2}$ such as:

$$
\left\{\begin{array}{l}
A_{i}^{z_{i}^{x}+}=B_{j}^{z_{j}^{x}+}=x, \\
A_{i}^{z_{i}^{x}-} \leq B_{j}^{z_{j}^{x}-} .
\end{array}\right.
$$

\footnotetext{
${ }^{1}$ proof is not given here due to lack of space
} 
To outline existing partial conflict $k_{i, j}$ between the agents $\mathcal{E}_{i}$ and $\mathcal{E}_{j}[6]$, the $z$ label values of the modes are denoted by $K_{i}$ and $K_{j}$ :

$$
\left\{\begin{array}{l}
K_{i}=\frac{\left|\mu_{j}-\mu_{i}\right|}{\sigma_{i}}, \\
K_{j}=\frac{\left|\mu_{j}-\mu_{i}\right|}{\sigma_{j}} .
\end{array}\right.
$$

Relations (5) show that the absolute value of line directions of (1), (2) and (3) are equal to $\left|\operatorname{arctg}\left(\frac{\sigma_{i}}{\sigma_{j}}\right)\right|$ and correspond to angles $\alpha_{1}, \alpha_{2}$ and $\alpha_{3}$ in figure 2(a).

\subsection{Focal intervals intersection or union overview}

As shown in figure 2(b), focal intervals $A_{i}^{z_{k}} \in \mathcal{F}_{i}$ and $B_{j}^{z_{l}} \in \mathcal{F}_{j}$ can directly be drawn on the chart by respectively vertical and horizontal line segments since (1), (2) and (3) correspond to the focal intervals bounds. The path defined by half-lines (1), (2) and (3) covers $\Omega$. It becomes thus easy to analyze pairs in $\mathcal{F}_{i} \times \mathcal{F}_{j}$ to deduce their intersection or union. For instance, intervals shown in figure $2(\mathrm{~b})$ are such as $A_{i}^{z_{k}} \cap B_{j}^{z_{l}}=\left[B_{j}^{z_{l}-}, A_{i}^{z_{k}+}\right]$ and $A_{i}^{z_{k}} \cup B_{j}^{z_{l}}=\left[A_{i}^{z_{k}-}, B_{j}^{z_{l}+}\right]$. When considering $z_{k}$ and $z_{l}$ respectively as horizontal and vertical cursors it is also possible to find the $z$ limits of intervals intersecting or including one another or not.

\subsection{Particular domains}

Figure 2(b) shows also that the (1), (2) and (3) lines separate $\mathcal{F}_{i} \times \mathcal{F}_{j}$ in four domains called $\mathcal{D}_{1}, \mathcal{D}_{2}, \mathcal{D}_{3}$ ans $\mathcal{D}_{4}{ }^{2}$. For $\mu_{i}<\mu_{j}, z_{i} \geq 0, z_{j} \geq 0$, defining pairs $\left(A_{i}^{z_{i}}, B_{j}^{z_{j}}\right) \in \mathcal{F}_{i} \times \mathcal{F}_{j}$, we have:

$$
\left\{\begin{array}{rlr}
z_{j}>K_{j}+\frac{\sigma_{i}}{\sigma_{j}} z_{i} & \Rightarrow A_{i}^{z_{i}} \subset B_{j}^{z_{j}} & \left(\mathcal{D}_{1}\right), \\
z_{j}<-K_{j}+\frac{\sigma_{i}}{\sigma_{j}} z_{i} & \Rightarrow A_{i}^{z_{i}} \supset B_{j}^{z_{j}} & \left(\mathcal{D}_{2}\right), \\
z_{j}<K_{j}+\frac{\sigma_{i}}{\sigma_{j}} z_{i}, & z_{j}>K_{j}-\frac{\sigma_{i}}{\sigma_{j}} z_{i}, \quad z_{j}>-K_{j}+\frac{\sigma_{i}}{\sigma_{j}} z_{i} & \\
& \Rightarrow A_{i}^{z_{i}} \cap B_{j}^{z_{j}} \notin\left\{\emptyset, A_{i}^{z_{i}}, B_{j}^{z_{j}}\right\} & \left(\mathcal{D}_{3}\right), \\
z_{j}<K_{j}-\frac{\sigma_{i}}{\sigma_{j}} z_{i}, & \Rightarrow A_{i}^{z_{i}} \cap B_{j}^{z_{j}}=\emptyset & \left(\mathcal{D}_{4}\right) .
\end{array}\right.
$$

When $\mu_{1}=\mu_{2}$ only $\mathcal{D}_{1}$ and $\mathcal{D}_{2}$ exist and are separated by the half-line $z_{2}=\frac{\sigma_{1}}{\sigma_{2}} z_{1}$.

2 proof is not given here due to lack of space 


\subsection{Consonant subsets of $\mathcal{F}_{i, j}$}

The focal set $\mathcal{F}_{i, j}$ obtained after a conjunctive or a disjunctive combination operation of $\mathcal{E}_{i}$ and $\mathcal{E}_{j}$ is composed of an infinite number of nested focal intervals subsets. These consonant subsets appear both in $\mathcal{D}_{1}, \mathcal{D}_{2}$ and $\mathcal{D}_{3}$ shown in figure 2(b) and are partially represented in figure 1(b) with the dark gray area. These subsets are not disjoint and consequently if the same interval belongs to several of them, it is necessary to integrate to get its total weight. The domain $\mathcal{D}_{4}$ differs from the other ones as it is empty in case of a conjunctive combination operation and composed of nested non convex intervals in case of a disjunctive one.

\section{Same length intervals resulting from intersection and union operations of focal ones}

\subsection{Intersection of focal intervals}

The length $l$ of the intersection of the pairs $\left(A_{i}^{z_{i}}, B_{j}^{z_{j}}\right) \in \mathcal{F}_{i} \times \mathcal{F}_{j}$ can be deduced from the characteristics of $\mathcal{D}_{1}, \mathcal{D}_{2}, \mathcal{D}_{3}$ and $\mathcal{D}_{4}$ given by relations (10). $l$ only depends on the pdfs bet $f_{i}$ and $\operatorname{bet} f_{j}$ parameters and the focal intervals indexes $z_{i}$ and $z_{j}$ :

$$
\begin{cases}\mathcal{D}_{1}: A_{i}^{z_{i}} \cap B_{j}^{z_{j}}=A_{i}^{z_{i},}, & l\left(A_{i}^{z_{i}} \cap B_{j}^{z_{j}}\right)=2 \sigma_{i} z_{i}, \\ \mathcal{D}_{2}: A_{i}^{z_{i}} \cap B_{j}^{z_{j}}=B_{j}^{z_{j}}, & l\left(A_{i}^{z_{i}} \cap B_{j}^{z_{j}}\right)=2 \sigma_{j} z_{j}, \\ \mathcal{D}_{3}: A_{i}^{z_{i}} \cap B_{j}^{z_{j}}=\left[B_{j}^{z_{j}-}, A_{i}^{z_{i}}\right], & l\left(A_{i}^{z_{i}} \cap B_{z_{j}}^{z_{j}}\right)=-\left|\mu_{2}-\mu_{1}\right|+\sigma_{i} z_{i}+\sigma_{j} z_{j}, \\ \mathcal{D}_{4}: A_{i}^{z_{i}} \cap B_{j}^{z_{j}}=\emptyset, & l\left(A_{i}^{z_{i}} \cap B_{j}^{z_{j}}\right)=0 .\end{cases}
$$

We can find the elements of $\mathcal{F}_{i} \times \mathcal{F}_{j}$ having an identical intersection length $L=l\left(A_{i}^{z_{i}} \cap B_{j}^{z_{j}}\right)$ with the help of relations (11). For instance, the $z$ indexes of these elements in $D_{3}$ are:

$$
\sigma_{j} z_{j}=L+\left|\mu_{2}-\mu_{1}\right|-\sigma_{i} z_{i}
$$

thus:

$$
z_{j}=A-\frac{\sigma_{i}}{\sigma_{j}} z_{i} \text { with } A=\frac{L}{\sigma_{j}}+K_{j} .
$$

This is graphically represented in figure $2(\mathrm{c})$ by the line segment (6) bounded by points $(F, C)$ and $(D, E)$ and crossing the point $\left(z_{i}, z_{j}\right)=(0, A)$ with $A$ as defined in relation (13). Pairs $\left(A_{i}^{z_{i}}, B_{j}^{z_{j}}\right)$ in $\mathcal{D}_{1}$ having also an intersection length equal to $L$ correspond to the points of the half-line (5) crossing $(F, C)$ since $A_{i}^{z_{i}} \cap B_{j}^{z_{j}}=A_{i}^{z_{i}}$ in $\mathcal{D}_{1}$. Pairs of intervals on the half line (7) crossing $(D, E)$ in figure 2(c) have an intersection length equal to $L$ too since $A_{i}^{z_{i}} \cap$ 
$B_{j}^{z_{j}}=B_{j}^{z_{j}}$ in $\mathcal{D}_{2}$.

At last, values $A$ to $F$ are quite interdependent ${ }^{3}$ depending on $K_{i}$ and $K_{j}$ values. This is due to the symmetry properties of straight lines (1) to (7) in figures 2(b) and 2(c). Many relations depending on these parameters lead to the value of $L$ such as $L=2 \sigma_{j}\left(C-K_{j}\right)=2 \sigma_{i}\left(D-K_{i}\right)$ for instance.

\subsection{Union of focal intervals}

One can deduce from the relations (10) related to the domains $\mathcal{D}_{1}, \mathcal{D}_{2}, \mathcal{D}_{3}, \mathcal{D}_{4}$ that for the union operation of pairs $\left(A_{i}^{z_{i}}, B_{j}^{z_{j}}\right) \in \mathcal{F}_{i} \times \mathcal{F}_{j}$, we have:

$$
\left\{\begin{array}{lll}
\mathcal{D}_{1}: A_{i}^{z_{i}} \cup B_{j}^{z_{j}}=B_{j}^{z_{j}}, & l\left(A_{i}^{z_{i}} \cup B_{j}^{z_{j}}\right)=2 \sigma_{j} z_{j}, \\
\mathcal{D}_{2}: A_{i}^{z_{i}} \cup B_{j}^{z_{j}}=A_{i}^{z_{i}}, & l\left(A_{i}^{z_{i}} \cup B_{j}^{z_{j}}\right)=2 \sigma_{i} z_{i}, \\
\mathcal{D}_{3}: A_{i}^{z_{i}} \cup B_{j}^{z_{j}}=\left[B_{j}^{z_{j}-}, A_{i}^{z_{i}+}\right], & l\left(A_{i}^{z_{i}} \cup B_{j}^{z_{j}}\right)=\left|\mu_{2}-\mu_{1}\right|+\sigma_{i} z_{i}+\sigma_{j} z_{j}, \\
\mathcal{D}_{4}: A_{i}^{z_{i}} \cap B_{j}^{z_{j}}=\emptyset, & l\left(A_{i}^{z_{i}} \cup B_{j}^{z_{j}}\right)=2\left(\sigma_{i} z_{i}+\sigma_{j} z_{j}\right) .
\end{array}\right.
$$

As expressed in (14), concerning $\mathcal{D}_{3}$, one can write:

$$
l\left(A_{i}^{z_{i}} \cup B_{j}^{z_{j}}\right)-\left|\mu_{2}-\mu_{1}\right|=\sigma_{i} z_{i}+\sigma_{j} z_{j} .
$$

From (15), pairs $\left(A_{i}^{z_{i}}, B_{j}^{z_{j}}\right) \in \mathcal{D}_{3}$ having a constant union length $L=l\left(A_{i}^{z_{i}} \cup\right.$ $\left.B_{j}^{z_{j}}\right)$ satisfy thus:

$$
z_{j}=A-\frac{\sigma_{i}}{\sigma_{j}} z_{i} \text { with } A=\frac{L}{\sigma_{j}}-K_{j} .
$$

This relation corresponds in figure $2(\mathrm{~d})$ to the line segment (6) bounded by points $(F, C)$ and $(D, E)$ and crossing the point $\left(z_{i}, z_{j}\right)=(0, A)$ with $A$ as defined in relation (16). Pairs in $\mathcal{D}_{1}$ having a union length equal to $L$ correspond to the points of the line segment (8). This is also the case in $\mathcal{D}_{2}$ for the points of the line segment (9.). As in the case of intersection operation, many relations ${ }^{4}$ link the value of $L$ to the parameters of pdfs bet $f_{i}$ and bet $f_{j}$. For instance we have $L=2 \sigma_{j} C=2 \sigma_{i} D$.

\section{Conclusion and Acknowledgment}

As we have seen, the focal set $\mathcal{F}_{i, j}$ obtained in case of a conjunctive (resp. disjunctive) combination of two pieces of evidence $\mathcal{E}_{i}$ and $\mathcal{E}_{j}$ with consonant

\footnotetext{
${ }^{3}$ proof is not given here due to lack of space
}

${ }^{4}$ proof is not given here due to lack of space 


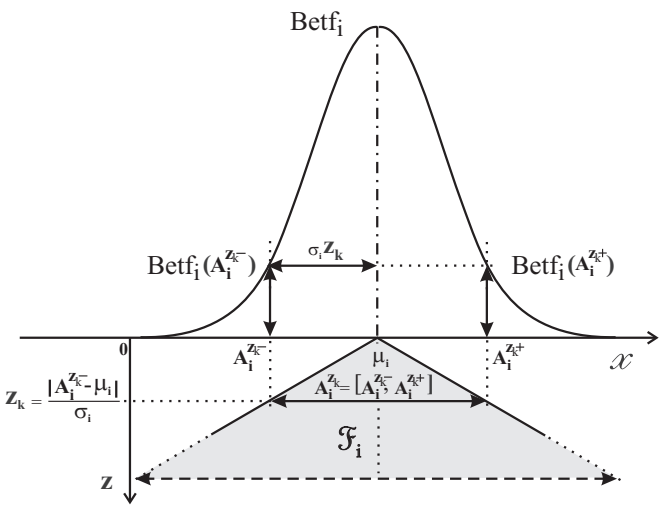

(a) Focal intervals domain $\mathcal{F}_{i}$ resulting from a Gaussian pdf.

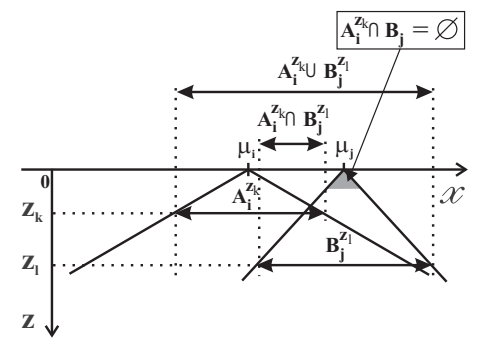

(b) Intersection and union of focal intervals resulting from two Gaussian pdfs.

Fig. 1 Focal intervals graphical representation relatively to the $z$ label value.

focal domains is not as heterogeneous as it seems to be. Intervals belonging to $\mathcal{F}_{i, j}$ are sorted into only four domains. In each of these domains, pairs $\left(A_{i} \in \mathcal{F}_{i}, B_{j} \in \mathcal{F}_{j}\right)$ of focal intervals share common properties regarding intersection $A_{i} \cap B_{j}$ and union $A_{i} \cup B_{j}$. These four domains can be graphically represented in a linear space where they are separated by straight lines when the focal sets $\mathcal{F}_{i}$ and $\mathcal{F}_{j}$ are composed of centered and consonant intervals. At last, elements of $\mathcal{F}_{i, j}$ having a same length are linked by linear relations. This can be useful in problems where interval lengths have to be taken into account.

Authors are indebted to J. Klein for the review of this work.

\section{References}

1. Caron, F., Ristic, B., Duflos, E., Vanheeghe, P.: Least commited basic belief density induced by a multivariate gaussian: formulation with applications. International Journal on Approximate Reasoning, vol. 48, no. 2, 419-436 (2008)

2. Doré P.-E., Martin A., Khenchaf A.: Constructing of least commited basic belief density linked to a multimodal probability density, in COGIS, Paris (Fr), 2009.

3. Ristic, B., Smets,P.: Belief function theory on the continuous space with an application to model based classification. In IPMU'04, Information Processing and Management of Uncertainty in Knowledge Based Systems, pp. 1119-1126, Paris (Fr) (2004)

4. Smets, P.: Belief functions on real numbers. International Journal of Approximate Reasoning, vol. 40, no. 3, 181-223 (2005) 
5. Strat, T.H.: Continuous belief functions for evidential reasoning, in AAAI-84 (Ed., National Conference on Artificial Intelligence, 308-313, (1984)

6. Vannobel, J.-M.: Continuous belief functions: singletons plausibility function in conjunctive and disjunctive combination operations of consonant bbds. Proceedings of Workshop on the theory of belief functions, CDROM, 6 pages. Brest(Fr) (2010)

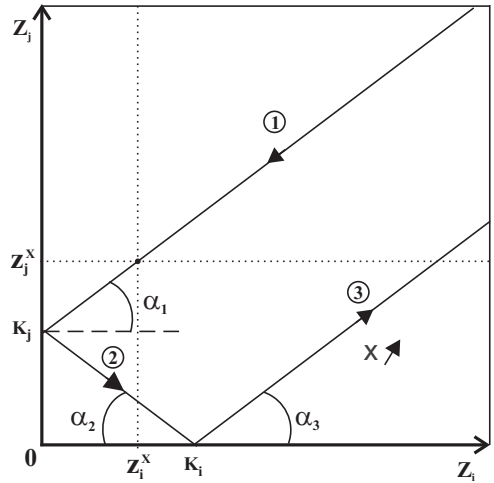

(a) Pairs of intervals having a common lower or upper bound

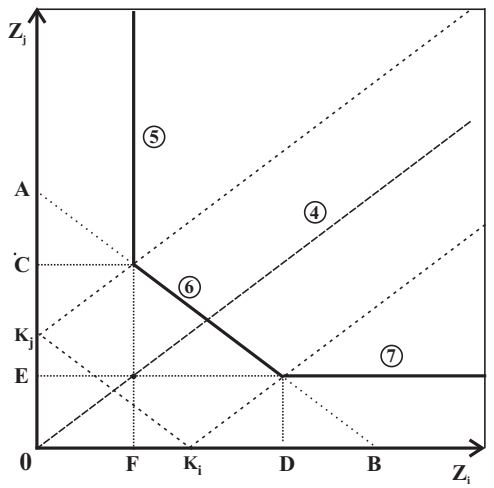

(c) Same length intervals resulting from a conjunctive combination

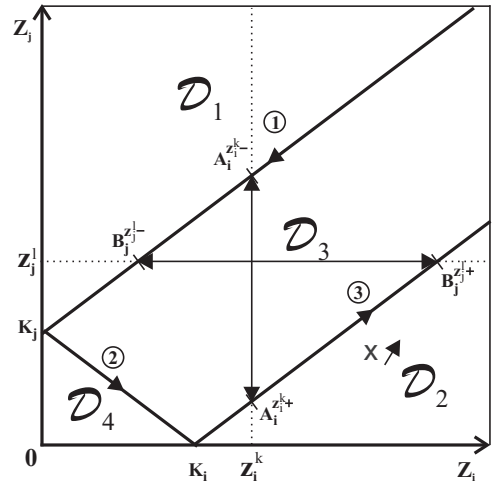

(b) Domains of identical properties

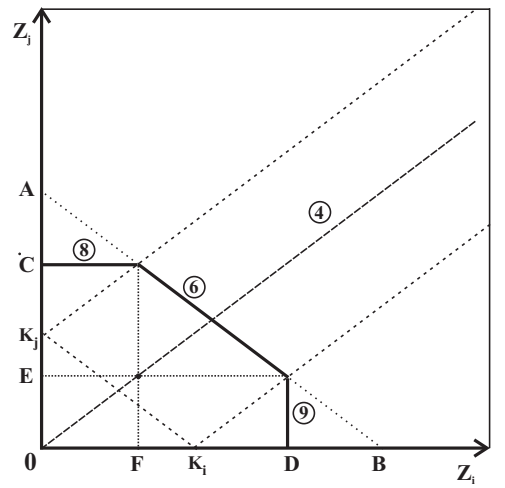

(d) Same length intervals resulting from a disjunctive combination

Fig. 2 Graphical representations of focal intervals properties. 